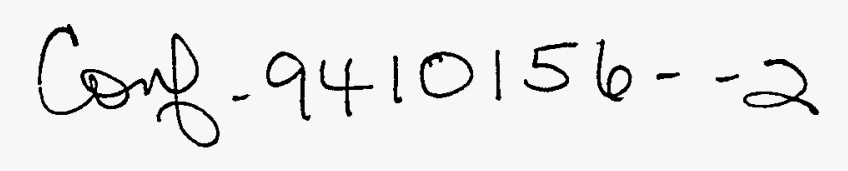

\title{
SAN094-1375C \\ Synthetic Aperture Radar Processing with Polar Formatted Subapertures
}

\author{
Armin W. Doerry
}

Sandia National Laboratories, Albuquerque, New Mexico

\begin{abstract}
Synthetic Aperture Radar (SAR) uses the motion of a small real antenna to synthesize a larger aperture, and thereby achieve very fine azimuth resolution. Efficient $S A R$ image formation requires modelling the radar echo and compensating (focusing) the delay and phase for various positions in the target scene. Polar-Format processing is one successful algorithm developed to process large scenes at fine resolutions, but is still limited, especially at resolutions near a wavelength. This paper shows how using tiers of subapertures can overcome the limitations of Polar-Format processing and increase the focused scene size substantially while using only efficient vector multiplies and Fast Fourier Transforms.
\end{abstract}

\section{1: Introduction and summary}

The motion of a radar antenna relative to a target introduces a delay and phase in the echo that is a function of the antenna motion. When the antenna motion traces an arc, and the radar echoes are coherently collected, the signal data may be digitally processed to achieve an azimuth resolution much finer than that capable by the real antenna alone. In this way a virtual antenna array can be synthesized that may be many meters (or even kilometers) long. This technique is known as Synthetic Aperture Radar (SAR) processing, and is illustrated in figure 1. The distance function from each point on the aperture to each location in the scene being imaged is unique, that is, different for each location in the scene. Efficient processing of the data into a SAR image, however, necessitates using transform techniques which treat areas of the scene identically. The concept is to 'focus' to a scene center and use a transform such as the 2dimensional Discrete Fourier Transform (DFT) to form an image of the area around the scene center. Locations not at the scene center will appear to move or 'migrate' around the scene center as the synthetic aperture is traced, thereby
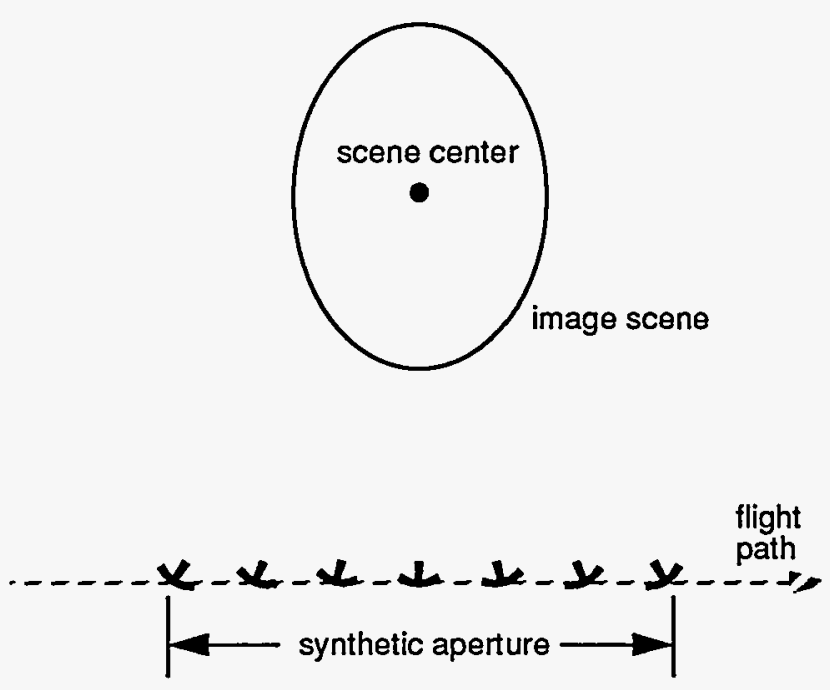

Figure 1: SAR Concept

smearing their response, and also suffer phase variations (defocusing) relative to the scene center. These effects worsen with distance from the scene center. After transformation into an image, the result is an image with worsening impulse response (broadening point spread function) as distance from the scene center increases. The degree of tolerance for image quality degradation at the scene edges limits the focused 'scene size'. The degradation, and hence scene size, is strongly affected by operating frequency and the resolution sought, with finer resolutions (longer synthetic apertures) and lower frequencies allowing smaller focused scene sizes [1]. The challenge for image formation algorithms is to focus a large scene at low frequencies and fine resolutions.

A number of image formation algorithms using various signal processing techniques have been proposed with various degrees of effectiveness in the low-frequency, fine resolution case. For airborne SAR, with its typically notvery-straight flight paths, a most effective technique is Polar-Format processing, first proposed by Walker [6]. But spatially variant phase errors limit the focused scene 


\section{DISCLAIMER}

This report was prepared as an account of work sponsored by an agency of the United States Government. Neither the United States Government nor any agency thereof, nor any of their employees, make any warranty, express or implied, or assumes any legal liability or responsibility for the accuracy, completeness, or usefulness of any information, apparatus, product, or process disclosed, or represents that its use would not infringe privately owned rights. Reference herein to any specific commercial product, process, or service by trade name, trademark, manufacturer, or otherwise does not necessarily constitute or imply its endorsement, recommendation, or favoring by the United States Government or any agency thereof. The views and opinions of authors expressed herein do not necessarily state or reflect those of the United States Government or any agency thereof. 


\section{DISCLAIMER}

Portions of this document may be illegible in electronic image products. Images are produced from the best available original document. 
diameter $D$ to

$$
D \leq 4 \rho_{x}\left(\left\|\mathbf{r}_{c 0}\right\| / \lambda_{0}\right)^{1 / 2}
$$

where $\rho_{\mathrm{x}}$ is the azimuth resolution, $\left\|\mathbf{r}_{c 0}\right\|$ is the nominal range, and $\lambda_{0}$ is the nominal radar wavelength.

One proposed solution is to divide an aperture into subapertures (sub-arrays) [2,5], and perhaps those subapertures into subsubapertures, and so on, in a tiered fashion to allow compensation of the phase errors that limit Polar-Format processing. Each subaperture would by itself be used to form a coarse resolution image using Polar-Format processing. The information extracted from the coarse resolution images is used to estimate appropriate phase corrections, whereupon processing occurs across co-located pixels in each of the coarse resolution images, again using Polar-Format processing, to form a finer resolution image, and so on. These stages form a pipeline architecture that uses only easily implemented vector multiplies and DFTs, to overcome the scene size barrier in equation (1). This paper shows how the new limit for scene size for this technique using $N_{\mathrm{s}}$ levels (tiers) of subapertures becomes

$$
D \leq 4 \rho_{x}\left(\left\|r_{c 0}\right\| / \lambda_{0}\right)^{\frac{\left(N_{s}+1\right)}{\left(N_{s}+2\right)}} \text {. }
$$

\section{2: One-dimensional analogy}

Consider a function of the form

$$
f(n)=\exp j\left(\omega n+a \omega^{2} n^{2}\right),
$$

with known constant $a, 0<a \ll 1$, unknown constant $\omega$, $-\pi / 2 \leq-\Omega / 2 \leq \omega \leq \Omega / 2<\pi / 2$, and series index $n$, available over an aperture $N / 2 \leq n \leq N / 2-1$. The challenge is to determine $\omega$ with as fine a precision and accuracy as possible within the range $|\omega| \leq \Omega / 2$, and efficiently.

If $a$ were zero, the task would simply amount to a Discrete Fourier Transform (DFT) across $n$ and yield a resolution $\rho$ in $\omega$ of about $2 \pi / N$. For this case, larger $N$ means finer resolution, without bound.

For nonzero $a$, we must consider the quadratic phase term (quadratic in $n$ ). For small $\Omega$ and small $N$, this term can be ignored. A reasonable criteria might be

$$
a(\Omega / 2)^{2}(N / 2)^{2} \leq \pi / 2 .
$$

Performing an FFT across the data set limited by equation (4) then yields the constraint

$$
\Omega \leq \rho / \sqrt{\pi a / 2} \text {. }
$$

Here we see the basic dilemma. For using the DFT, or its efficient version, the Fast Fourier Transform (FFT), the larger $\Omega$ that we are interested in, the coarser the resolution is with which we can identify $\omega$. The challenge becomes to exceed the limit in equation (5). To do this we need to mitigate the effects of the quadratic phase term which is an error term for FFT processing.

\section{3: Subapertures}

We do this by making a coarse resolution estimate of $\omega$, and use this to compensate $f(n)$ before proceeding to a fine resolution estimate. Conceptually, we make coarse resolution estimates of $\omega$ by dividing the aperture into subapertures, as in figure 2(b). Mathematically, we do this by splitting the domain of index $n$ into groups of indices $m_{1}$ and $m_{2}$, by making $n=m_{1}+\Delta_{2} m_{2}$, where $m_{1}$ is intra-subaperture index and is limited to the domain $-M_{1} / 2 \leq m_{1} \leq M_{1} / 2-1, m_{2}$ is the inter-subaperture index and is limited to the domain $-M_{2} / 2 \leq m_{2} \leq M_{2} / 2-1$, and $\Delta_{2}$ is a data decimation factor, with subapertures overlapped by an amount $\left(M_{1}-\Delta_{2}\right)$. Overlapping subapertures is necessary to control sidelobes. We rewrite the function $f(n)$ as

$$
\begin{aligned}
& f\left(m_{1}, m_{2}\right)= \\
& \quad \exp j\left(\omega\left(m_{1}+\Delta_{2} m_{2}\right)+a \omega^{2}\left(m_{1}+\Delta_{2} m_{2}\right)^{2}\right) .
\end{aligned}
$$

The plan now is to perform a DFT across a much shorter ranged index $m_{1}$, and do this for each index value $m_{2}$, but first we need to examine the quadratic phase term $a \omega m_{1}$. To keep this term's effects negligible, we choose $M_{1}$ such that

$$
a(\Omega / 2)^{2}\left(M_{1} / 2\right)^{2} \leq \pi / 2 .
$$

With this restriction, a DFT of equation (6) yields approximately

(a) One large aperture

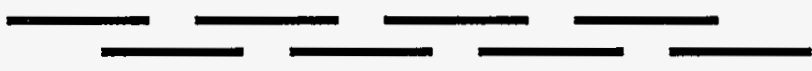

(b) Aperture divided into one level of subapertures

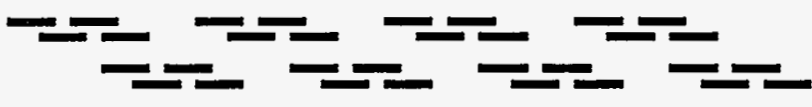

(c) Aperture divided into two levels of subapertures

Figure 2. Aperture divided into subapertures, and subsubapertures 


$$
\begin{aligned}
& f_{2}\left(u_{1}, m_{2}\right) \approx \\
& \quad \operatorname{csinc}_{M_{1}}\left[\frac{M_{1}}{2}\left(\omega+2 a \omega^{2} \Delta_{2} m_{2}-\frac{2 \pi u_{1}}{M_{1}}\right)\right] \\
& \quad \times \exp j\left(\omega \Delta_{2} m_{2}+a \omega^{2} \Delta_{2}^{2} m_{2}^{2}\right)
\end{aligned}
$$

where we define $\operatorname{csinc}_{\mathrm{M}}()$ as a convenient shorthand for the complex discrete sinc function, i.e.

$$
\operatorname{csinc}_{M}(x) \equiv \frac{\sin (x)}{\sin (x / M)} e^{-j x / M}
$$

The phase rotation in this function is typically negligible, especially in light of the amplitude envelope. In equation (8), the csinc function resolves $\omega$ with resolution $\left(2 \pi / M_{1}\right)$, and locates it with the estimate

$$
\hat{\omega} \approx 2 \pi u_{1} / M_{1}-2 a \hat{\omega}^{2} \Delta_{2} m_{2} .
$$

The problem here is that the estimate of $\omega$ depends on subaperture index value $m_{2}$, that is, the location of $\hat{\omega}$ 'migrates' with $m_{2}$. In fact, with large enough $m_{2}$, the migration will transcend several resolution cells (values of $\left.u_{1}\right)$. Therefor, for any one value of $u_{1}$, the migration has the effect of 'windowing' the data over index $m_{2}$, as the amplitude peak moves into and then out of the appropriate resolution cell $u_{1}$. Since we will ultimately want to perform a DFT across index $m_{2}$, it is desirable for the migration to be no more than about one coarse resolution cell $\rho_{1}$ in total, over all $m_{2}$ for any $\omega$. This constrains

$$
2 a\left(\frac{\Omega}{2}\right)^{2} \Delta_{2}\left(\frac{M_{2}}{2}\right) \leq \frac{1}{2}\left(\frac{2 \pi}{M_{1}}\right),
$$

which can be rewritten as,

$$
\rho_{1}=2 \pi / M_{1} \geq a \Omega^{2} \Delta_{2} M_{2} / 2 .
$$

Note that this is more severe than equation (7) since $\Delta_{2} M_{2} 》 M_{1} / 2$. With this constraint, migration can be ignored in equations (8) and (10), and allows us to compensate, or 'focus', equation (8) prior to a DFT across $m_{2}$, by performing the phase adjustment

$$
\begin{aligned}
f_{2}^{*}\left(u_{1}, m_{2}\right)= & f_{2}\left(u_{1}, m_{2}\right) \\
& \times \exp j\left(-\hat{\omega} \Delta_{2} m_{2}-a \hat{\omega}^{2} \Delta_{2}^{2} m_{2}^{2}\right),
\end{aligned}
$$

with the result expanded to

$$
\begin{aligned}
& f_{2}^{*}\left(u_{1}, m_{2}\right) \approx \operatorname{csinc}_{M_{1}}\left[\frac{M_{1}}{2}\left(\omega-\frac{2 \pi u_{1}}{M_{1}}\right)\right] \\
& \times \exp j\left((\omega-\hat{\omega}) \Delta_{2} m_{2}+a\left(\omega^{2}-\hat{\omega}^{2}\right) \Delta_{2}^{2} m_{2}^{2}\right) .
\end{aligned}
$$

By limiting

$$
a\left(\omega^{2}-\hat{\omega}^{2}\right)^{2} \Delta_{2}^{2}\left(\frac{M_{2}}{2}\right)^{2} \leq \frac{\pi}{2}
$$

for all $\omega$, a final DFT across $m_{2}$ yields approximately

$$
\begin{aligned}
f_{3}\left(u_{1}, u_{2}\right) \approx \operatorname{csinc}_{M_{1}}\left[\frac{M_{1}}{2}\left(\omega-\frac{2 \pi u_{1}}{M_{1}}\right)\right] \\
\times \operatorname{csinc}_{M_{2}}\left[\frac{M_{2} \Delta_{2}}{2}\left((\omega-\hat{\omega})-\frac{2 \pi u_{2}}{M_{2} \Delta_{2}}\right)\right] .
\end{aligned}
$$

The second, and final, best estimate of $\omega$ becomes

$$
\text { (2) } \hat{\omega} \approx \frac{2 \pi u_{1}}{M_{1}}+\frac{2 \pi u_{2}}{M_{2} \Delta_{2}}
$$

with a final resolution of $\rho_{2}=(2 \pi) /\left(\Delta_{2} M_{2}\right)$. A sufficient condition to meet the limit in equation (15) is

$$
a \rho_{1} \Omega \Delta_{2}^{2}\left(M_{2} / 2\right)^{2} \leq \pi / 2 .
$$

This, with the migration limit of equation (12), yields the ultimate constraint for a single level of subapertures as

$$
\Omega \leq \rho_{2} 2^{-1 / 3}(\pi a)^{-2 / 3},
$$

where $\rho_{2}=2 \pi /\left(\Delta_{2} M_{2}\right)$. Equation (19) represents an improvement over equation (5) for $a<1 /(32 \pi)$. The benefit of subapertures is thus established.

A similar development for two tiers of subapertures, illustrated in figure $2(\mathrm{c})$, yields an improved constraint

$$
\Omega \leq \rho_{3} 2^{-3 / 4}(\pi a)^{-3 / 4} \text {. }
$$

In fact, for $N_{\mathrm{s}}$ tiers of subapertures the ultimate constraint relating resolution and frequency range can be generalized to

$$
\Omega \leq \rho_{\left(N_{s}+1\right)} 2^{\frac{-\left(2 N_{s}-1\right)}{\left(N_{s}+2\right)}}(\pi a)^{\frac{-\left(N_{s}+1\right)}{\left(N_{s}+2\right)}}
$$

Each additional tier of subapertures improves the constraint, but with diminishing returns. The benefit is derived from the operation in equation (13), namely, the compensation of error terms with a coarse resolution estimate of $\hat{\omega}$. The use of subapertures is constrained by limiting migration to acceptable levels, usually about one current resolution cell over the entire aperture.

\section{4: Polar-format SAR processing limits}

Consider a linear-FM chirp radar, collecting echo samples along a flight path at positions indexed by $n$. $N / 2 \leq n \leq N / 2-1$, with geometry defined in figure 3 . The echoes are deramped and sampled at relative times indexed by $i, I / 2 \leq i \leq I / 2-1$. The radar operates with 


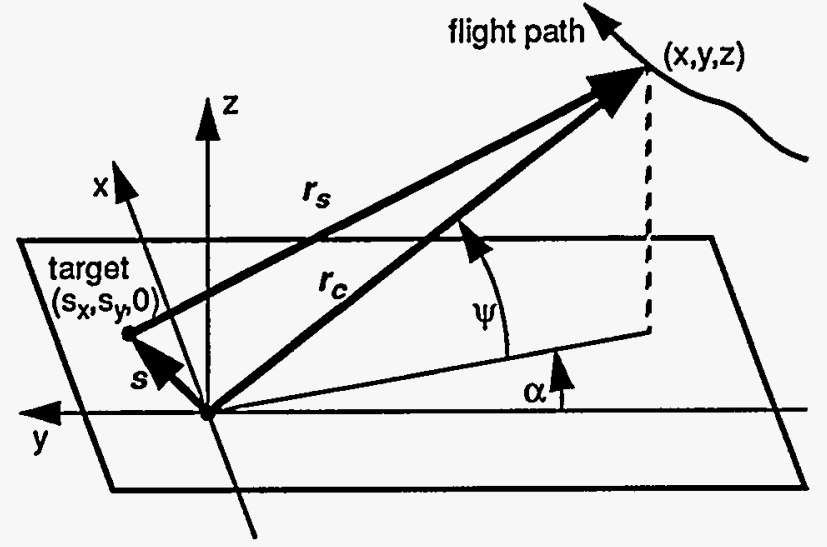

Figure 3. SAR geometry.

center frequency $\omega_{n}$, chirp rate $\gamma_{n}$, and ADC sample period $\mathrm{T}_{\mathrm{s}}$. With sufficiently long pulses [3], the radar's sampled video signal can be described by

$$
\begin{array}{r}
X_{V}(i, n) \approx A\left(r_{s}\right) \exp j\left\{\frac{2}{c}\left[\omega_{n}+\gamma_{n} T s\right] \cos \psi_{n} \cos \alpha_{n}\right. \\
\left.\times\left(s_{x} \tan \alpha_{n}-s_{y}+\xi_{r}(n)\right)\right\},
\end{array}
$$

where $A\left(r_{s}\right)$ represents the amplitude of the echo from the target. The function $\xi_{r}$ represents spatially variant error terms that can be described with a polynomial in $n$. By adjusting $\omega_{n}$ and $\gamma_{n}$ to compensate for variations in $\cos \psi_{n}$ and $\cos \alpha_{n}$, and sampling at equal increments in $\tan \alpha_{n}$, equation (22) can be simplified to

$$
\begin{aligned}
& X_{V}(i, n) \approx A\left(r_{s}\right) \\
& \times \exp \left\{\frac{2 \omega_{0} \cos \psi_{0}}{c}\left(1+\frac{\gamma_{0} T_{s}}{\omega_{0}} i\right)\left(s_{x} n-s_{y}+\xi_{r}(n)\right)\right\},
\end{aligned}
$$

The processing strategy is then to resample along $n$ as a function of $\left(1+\left(\gamma_{0} T_{s} i\right) / \omega_{0}\right)$, followed by a 2dimensional DFT over $i$ and the resampled $n$. This can be accomplished by employing a chirp-Z transform (CZT) over $n$ that incorporates the resampling, followed by a FFT over $i$ [4]. Recall that the CZT can be implemented with FFTs and vector multiplies. By limiting the largest quadratic term in $\xi_{r}$ to less than $\pi / 2$, then processing in this manner will limit an individual scene to diameter $D$ such that

$$
D \leq 4 \rho_{x}\left(\left\|\mathbf{r}_{c 0}\right\| / \lambda_{0}\right)^{1 / 2},
$$

where $\rho_{x}$ is the nominal resolution in the $x$-direction at the scene center, $\left\|r_{c o}\right\|$ is the nominal range to the scene center, and $\lambda_{0}$ is the nominal wavelength of the radar, $\lambda_{0}=2 \pi c / \omega_{0}$.

\section{5: Polar Formatted Subapertures}

Now consider equation (23) where both indices $i$ and $n$ are each divided into a single tier of subapertures, as in figure 2(b), and analogous to equation (6).

$$
\begin{aligned}
& n=m_{1}+\Delta_{2} m_{2} \\
& i=k_{1}+\mu_{2} k_{2}
\end{aligned}
$$

Equation (23) then becomes

$$
\begin{aligned}
& X_{V}\left(k_{1}, k_{2}, m_{1}, m_{2}\right) \approx A\left(r_{s}\right) \\
& \quad \times \operatorname{expj}\left\{\frac{2 \omega_{0} \cos \Psi_{0}}{c}\left(1+\frac{\gamma_{0} T_{s} \mu_{2}}{\omega_{0}} k_{2}+\frac{\gamma_{0} T_{s}}{\omega_{0}} k_{1}\right)\right. \\
& \left.\quad \times\left(s_{x} \Delta_{2} m_{2}+s_{x} m_{1}-s_{y}+\xi_{r}\left(m_{1}+\Delta_{2} m_{2}\right)\right)\right\} .
\end{aligned}
$$

The processing strategy is straightforward and is illustrated in figure 4.

1. perform a CZT across $m_{1}$, adjusting output sample spacing as a function of $k_{1}$ and $k_{2}$. Use the resulting estimate of $s_{\mathrm{x}}$ to compensate the phase of the result.

2. perform a FFT across $k_{1}$. Use the result to optimally estimate $s_{\mathrm{x}}$ and $s_{\mathrm{y}}$ to further compensate the phase of the result.

3. perform a CZT across $m_{2}$, adjusting output sample spacing as a function of $k_{2}$. Use the result to again optimally estimate $s_{\mathrm{x}}$ and $s_{\mathrm{y}}$ to further compensate the phase of the result.

4. perform a FFT across $k_{2}$. The result is the complex SAR image.

Note that the error function $\xi_{r}$ formerly being a polynomial in $n$, now contains a number of cross product terms between $m_{1}$ and $m_{2}$. The cross terms that are linear coefficients of the index being transformed manifest

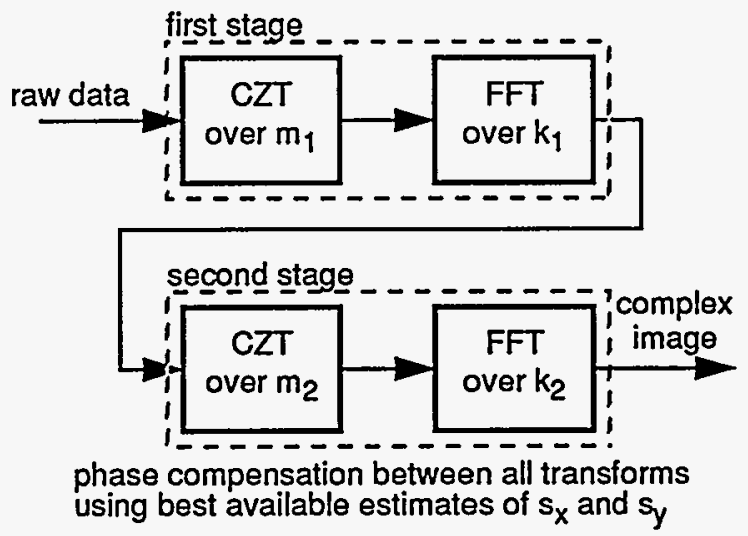

Figure 4. Processing strategy (1 tier) 
themselves as migration terms. Migration has the effect of windowing the data and thereby limiting the resolution of succeeding transforms. It is therefor necessary to limit migration to something on the order of one resolution cell across the entire aperture. This is done by limiting subaperture length, and hence subaperture resolution. Since migration affects succeeding transforms, resolution limits due to migration only affect the stages prior to the final transforms, and tend to dominate the effects of other error terms that broaden the impulse response. The final transform pair, however, are over data that contains no cross-product migration terms (or at least none that can't be accommodated by the final CZT) and therefor have resolutions limited by spatially variant phase errors, specifically the quadratic component of the error function.

Migration is limited to about one current (coarse) resolution cell with the following sufficient conditions,

$$
\begin{aligned}
& D_{x}^{2} \leq 8 \rho_{x, 1} \rho_{x, 2}\left(\left\|r_{c 0}\right\| / \lambda_{0}\right), \\
& D_{y}^{2} \leq 16 \rho_{x, 1} \rho_{x, 2}\left(\left\|r_{c 0}\right\| / \lambda_{0}\right),
\end{aligned}
$$

and

$$
\rho_{x, 1} \leq\left(4 / \lambda_{0}\right) \rho_{y, 1} \rho_{x, 2} \cos \psi_{0} .
$$

where $D_{\mathrm{x}}$ and $D_{\mathrm{y}}$ are the scene diameters in the $x$ and $y$ directions respectively, $\rho_{x_{1}, 1}$ and $\rho_{y_{1}, 1}$ are the resolutions in the $x$ and $y$ directions after the first CZT / FFT transform pair, and $\rho_{x, 2}$ and $\rho_{y, 2}$ are the resolutions in the $x$ and $y$ directions after the second and final CZT / FFT transform pair. We also define $\delta_{x, 1}$ and $\delta_{x, 2}$ as the first stage's pixel spacing in the $x$ and $y$ directions respectively.

Limiting the quadratic component of the spatially variant phase errors to about $\pi / 2$ limits the final stage CZT /FFT transform pair such that

$$
\delta_{x, 1} \leq\left(8 / D_{x}\right) \rho_{x, 2}^{2}\left(\left\|r_{c 0}\right\| / \lambda_{0}\right),
$$

and $\quad \delta_{y, 1} \leq\left(16 / D_{y}\right) \rho_{x, 2}^{2}\left(\left\|r_{c 0}\right\| / \lambda_{0}\right)$.

With the reasonable assumptions that $\rho_{x, 2} \geq \lambda_{0} / 2$, $\psi \leq \pi / 3$, and $\rho_{y, 1}=\rho_{x, 1}=\delta_{x, 1}=\delta_{y, 1}$, equations (28) thru (32) will allow an overall limit on scene diameter of

$$
D_{x^{\prime}} D_{y} \leq 4 \rho_{x, 2}\left(\left\|r_{c 0}\right\| / \lambda_{0}\right)^{2 / 3},
$$

which is clearly an improvement over the traditional limit given in equation (24). We also note that if the first stage oversamples its output $\left(\delta_{x, 1} \leq p_{x, 1}\right)$ then the scene diameter limit is further improved by a factor $\left(\rho_{x, 1} / \delta_{x, 1}\right)^{1 / 3}$. This is somewhat similar to the multiple corrections case described in [2].

Note that the initial sampling strategy along with the CZT make each stage a separate polar-format processed image formation step, but allows successive stages to

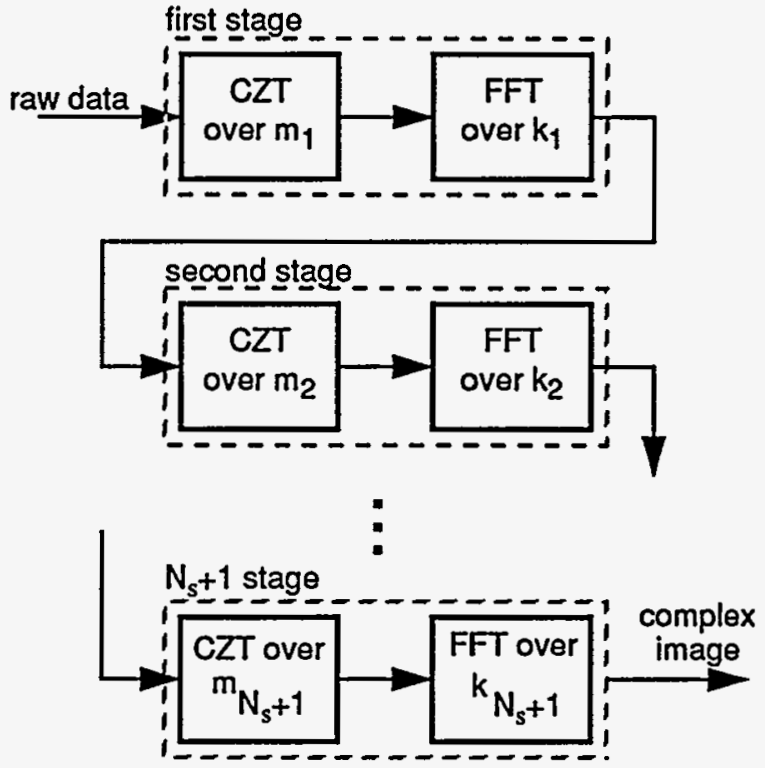

phase compensation between all transforms using best available estimates of $s_{x}$ and $s_{y}$

Figure 5. Processing strategy $\left(\mathrm{N}_{\mathrm{s}}\right.$ tiers)

achieve finer resolution. This strategy can be extended in a similar manner to an arbitrary number of tiers of subapertures. Any number $N_{\mathrm{s}}$ tiers will require $N_{\mathrm{s}}+1$ stages of FFT / CZT pairs, partitioned with indices

$$
\begin{aligned}
& n=m_{1}+\sum_{p=2}^{N_{s}+1}\left[m_{p} \prod_{q=2}^{p} \Delta_{q}\right], \\
& i=k_{1}+\sum_{p=2}^{N_{s}+1}\left[k_{p} \prod_{q=2}^{p} \mu_{q}\right] .
\end{aligned}
$$

The architecture for such a scheme is illustrated in figure 5. Equation (33) can then be extended to

$$
D_{x^{\prime}}, D_{y} \leq 4 \rho_{x_{s}\left(N_{s}+1\right)}\left(\left\|r_{c 0}\right\| / \lambda_{0}\right)^{\frac{\left(N_{s}+1\right)}{\left(N_{s}+2\right)}} .
$$

For scene diameters less than this limit, and particularly when image aspect ratios are non-unity, $D_{x} \neq D_{y}$, the assumption that each stage's output resolutions be equal in the $x$ and $y$ directions, $\rho_{y, n}=\rho_{x, n}$, may be relaxed, even to the point that a particular transform in some stage collapses to unity length, that is, vanishes. In any case, the relationship between intermediate resolutions need to be chosen to comply with the migration limitations, whereas the relationship between final resolutions need to concern themselves with spatially variant phase error limitations.

One useful derivative processing strategy is to achieve final $y$-resolution prior to the final CZT and final $x$ - 
resolution. This would allow the final CZT to compensate for geometric distortions at the edges of the scene, thereby allowing easier mosaicking of scenes from adjacent apertures into image strips [2].

\section{6: Examples}

Consider a SAR operating at $150 \mathrm{MHz}$, at a nominal elevation angle $\psi_{0}=30^{\circ}$, and a nominal range $\left\|r_{c 0}\right\|=5 \mathrm{~km}$. Figure 6 illustrates scene diameter limits vs. resolution for processing strategies employing various numbers of tiers of subapertures using equation (36).

The benefit of tiers of subapertures is also illustrated in figure 7, which compares impulse responses of simulated point targets located at various distances from the scene center along the $x$ axis. The data was processed to 1 meter resolution with a $\mathbf{- 3 5} \mathrm{dB}$ Taylor window. Other parameters are the same as for figure 6 .

\section{7: References}

[1] Ausherman, Dale A., Adam Kozma, Jack L. Walker, Harrison M. Jones, Enrico C. Poggio, "Developments in Radar Imaging", IEEE Transactions on Aerospace and Electronic Systems, Vol. AES-20, No. 4, July 1984, pp 363. 398.

[2] Burns, B. L., J. T. Cordaro, "SAR image formation algorithm that compensates for the spatially variant effects of antenna motion", SPIE Proceedings Vol 2230, SPIE's Intemational Symposium on Optical Engineering in Aerospace Sensing, Orlando, 4-8 April 1994.

[3] Doerry, A. W., "Patch Diameter Limitations due to High Chirp Rates in Focused Synthetic Aperture Radar Images", to be published in IEEE Transactions on Aerospace and Electronic Systems, tentatively scheduled for AES-30, No. 4, October 1994.

[4] Lawton, Wayne, "A New Polar Fourier Transform for Computer-Aided Tomography and Spotlight Synthetic Aperture Radar", IEEE Transactions on Acoustics, Speech, and Signal Processing, Vol. 36, No. 6, June 1988, pp 931933.

[5] Perry, R. P., R. C. DiPietro, A. Kozma, J.J. Vaccaro, "SAR Image formation processing using planar subarrays", SPIE Proceedings Vol 2230, SPIE's International Symposium on Optical Engineering in Aerospace Sensing, Orlando, 4-8 April 1994.

[6] Walker, Jack L., "Range-Doppler Imaging of Rotating Objects", IEEE Transactions on Aerospace and Electronic Systems, Vol. AES-16, No. 1, January 1980, pp 23-51.

\section{8: Acknowledgments}

This work performed at Sandia National Laboratories is supported by the U.S. Department of Energy under contract DE-AC04-94AL85000.

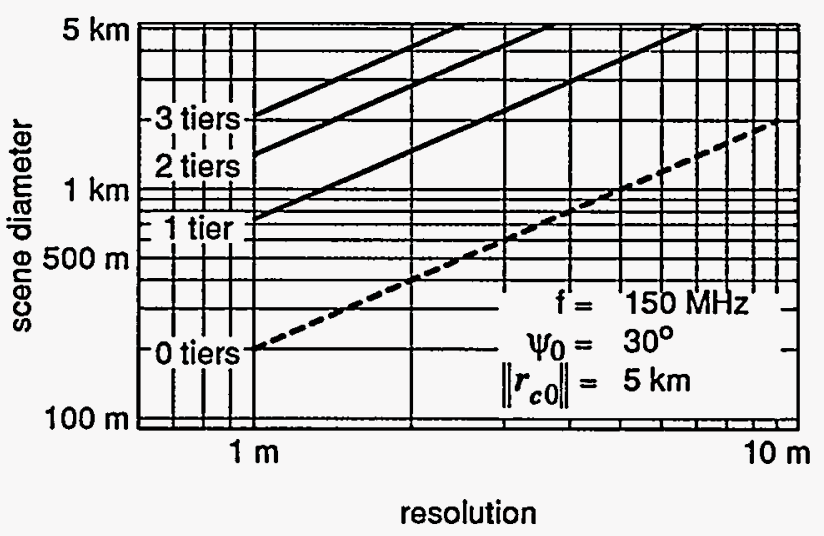

Figure 6. Scene diameter limits vs. resolution
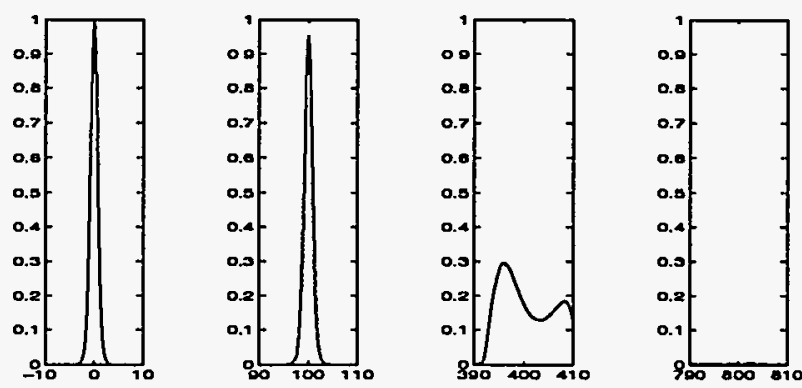

(a) 0 tiers - polar format processing
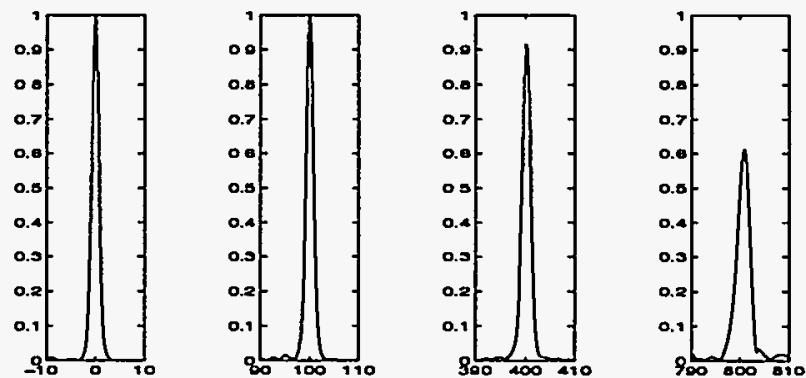

(b) 1 tier
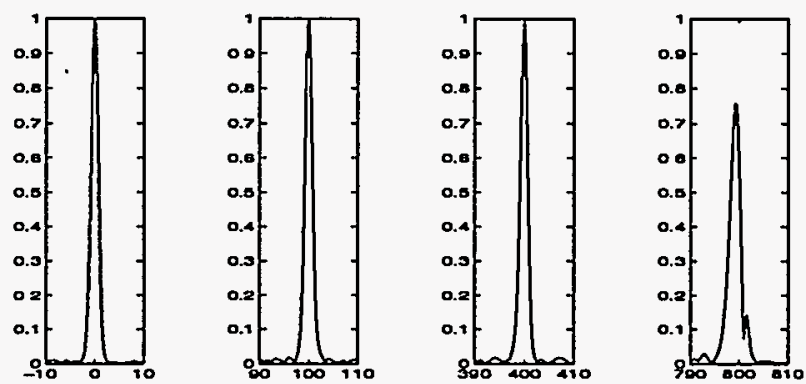

(c) 2 tiers

Targets at $0,100,400$, and $800 \mathrm{~m}$ from scene center

Figure 7. Point target response comparison 